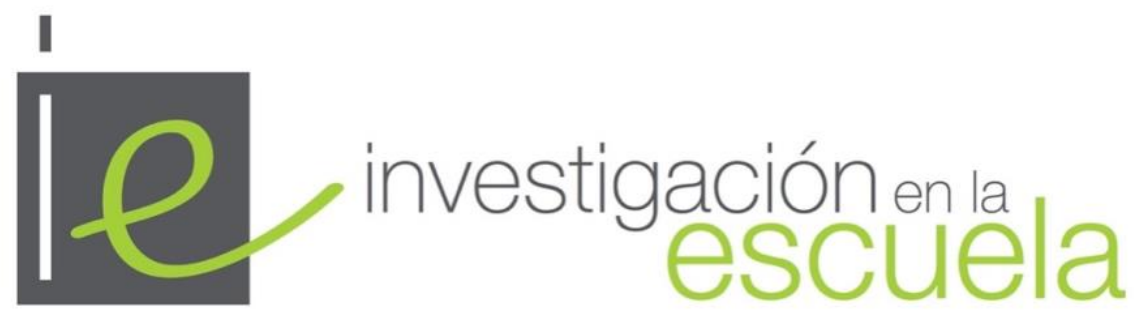

Revista de Investigación e Innovación Educativa nº 102, 2020 | e-ISSN 2443-9991

\title{
Emociones de estudiantes preuniversitarios en Ciencias Sociales con experiencias de gamificación
}

\author{
Emotions of pre-university students in Social Sciences with gamification experiences
}

iD Dr. Mario Corrales Serrano es Profesor de Educación Secundaria en Colegio Santa María Assumpta (España) mcorraletj@alumnos.unex.es·https://orcid.org/0000-0001-8520-9222

\section{Cómo citar este artículo}

Corrales Serrano, M. (2020). Emociones de estudiantes preuniversitario en Ciencias Sociales con experiencias de gamificación. Investigación en la Escuela, 102, 84-96. doi: http://doi.org/10.12795/IE.2020.i102.06

Resumen. El papel de las emociones en los procesos de enseñanza-aprendizaje está adquiriendo mucha relevancia en la investigación educativa de los últimos años. Sin embargo, en el área de ciencias sociales, no son muchos los estudios que se han llevado a cabo a este respecto. El presente trabajo es un análisis de la incidencia de las emociones en los procesos de enseñanza-aprendizaje de las ciencias sociales en experiencias didácticas que se han diseñado con metodología de gamificación. El objetivo principal es valorar la incidencia de esta metodología en la generación de emociones de cara al aprendizaje. La experiencia se ha llevado a cabo con 280 estudiantes de ESO y Bachillerato en materias de ciencias sociales. Para analizar los resultados se ha empleado una metodología de tipo cualitativo. Se han analizado fragmentos del diario de aprendizaje, en los que los estudiantes de la muestra se expresan acerca de cómo han vivido las experiencias didácticas de gamificación. El análisis se ha realizado mediante el software WebQDA, que permite extraer conclusiones del análisis de fuentes textuales. Los resultados apuntan a que, por un lado, hay una incidencia muy positiva de las emociones en las experiencias en las que se ha empleado la metodología de gamificación, aunque también se detecta que en algunos estudiantes se generan emociones que pueden complicar el aprendizaje.

\begin{abstract}
The role of emotions in the teaching-learning processes is acquiring much relevance in educational research in recent years. However, in the area of social sciences, not many studies have been carried out in this regard. The present work is an analysis of the incidence of emotions in the teaching-learning processes of the social sciences in didactic experiences that have been designed with gamification methodology. The main objective is to assess the incidence of this methodology in the generation of emotions for learning. The experience has been carried out with 280 ESO and Baccalaureate students in social sciences subjects. To analyse the results, a qualitative methodology has been used. Fragments of the learning diary have been analysed, in which the students in the sample express themselves about how they have lived didactic experiences of gamification. The analysis has been carried out using the WebQDA software, which allows conclusions to be drawn from the analysis of textual sources. The results suggest that, on the one hand, there is a very positive incidence of emotions in the experiences in which the gamification methodology has been used, although it is also detected that emotions are generated in some students that can complicate learning.
\end{abstract}

\section{Palabras clave $\cdot$ Keywords}

Emociones, gamificación, didáctica de las ciencias sociales, innovación, juegos digitales. Emotions, gamification, didactics of social sciences, innovation, digital games.

\section{Introducción}

El estudio de las emociones que se genera en los procesos de enseñanza-aprendizaje está adquiriendo una gran relevancia en las últimas décadas (Borrachero, 2015). En los primeros años de la década de los 
2000, de un modo muy explícito, Hargreaves, (2003, p. 12) afirma que "las emociones están en el corazón de la enseñanza". Una de las claves por las que esta dimensión se ha integrado con tanta fuerza en el ámbito de la investigación educativa es el hecho de que las relaciones interpersonales tienen una importancia fundamental en todo proceso educativo:

El trabajo en la enseñanza está basado principalmente en las relaciones interpersonales con los estudiantes y con otros compañeros, por lo que las experiencias emocionales son permanentes. Enfado, alegría, ansiedad, afecto, preocupación, tristeza, frustración... son algunos de los sentimientos que día a día vive el profesor con mayor o menor intensidad y amplitud. (Brígido et al., 2009, p. 3).

Por este motivo, la investigación acerca de la incidencia de las emociones en los procesos de enseñanzaaprendizaje está presente desde hace unos años en los diversos ámbitos de la educación. Su valor reside en hacer visible un ámbito de la educación que puede determinar el éxito o el fracaso del aprendizaje, pero que habitualmente no está presente en los procesos de transmisión de conocimientos, en las actividades prácticas o en los procesos de evaluación.

El estudio de las emociones tiene protagonismo en todas las etapas del sistema educativo. Un acercamiento a la literatura científica (Mellado et al., 2014; Fernández-Berrocal, et al. 2017) de los últimos años permite observar la proliferación de estudios en todos los niveles y materias del sistema educativo:

- En las etapas de Educación Infantil y Primaria, se han llevado a cabo estudios de tipo sistemático acerca del papel que ocupan las emociones en el aprendizaje (Cassà, 2016; Bisquerra Alzina \& Hernández Paniello, 2017); por otro lado, se han desarrollado estudios vinculados con la aplicación de la inteligencia emocional al aprendizaje (Aguaded Gómez \& Pantoja Chaves, 2015), y también con algunas áreas concretas de conocimiento, como la educación física (Miralles Pascual et al., 2017).

- En la etapa de Educación Secundaria se ha abordado la cuestión de las emociones en relación con el rendimiento académico de los estudiantes (Usán Supervía \& Salavera Bordás, 2018; Broc Cavero, 2019), así como herramienta para potenciar la motivación en el aprendizaje (Domínguez et al., 2016). También se han puesto en marcha interesantes programas de educación emocional, que han reportado resultados interesantes (Sánchez Calleja et al., 2018), y en el ámbito de materias como al Educación Física (Cera et al., 2015) o las Ciencias (Borrachero, 2015).

- Otro ámbito en el de destacan las investigaciones en el campo de las emociones es el ámbito de la formación del profesorado de diversas materias y para diferentes especialidades, como se puede observar en diversos trabajo de investigación (Retana Alvarado, et al. 2017; Kelchtermans \& Deketelaere, 2016; (Sánchez-Álvarez et al., 2015).

- Este proceso lleva desarrollándose varias décadas (Bisquerra Alzina, 2005) y sigue teniendo una gran relevancia en la actualidad (Torrijos-Fincias et al., 2018)

Un ámbito en el que, sin embargo, no se han llevado a cabo muchos estudios acerca de las emociones y el proceso de enseñanza-aprendizaje es el de la enseñanza de las ciencias sociales. La vinculación de estos saberes con un modo de desarrollar el aprendizaje más teórico, y menos vinculado con experiencias de tipo práctico (Corrales et al., 2019), ha hecho que los estudios emocionales se centren más en aprendizajes de contenidos vinculados a los saberes de tipo experimental (Bolaños Florido, 2016; Vercher Ferrándiz, 2019).

Vinculados al área de ciencias sociales, se han realizado también algunos estudios emocionales vinculados al patrimonio histórico (Cuenca-López y Estepa-Giménez, 2017; Gómez-Hurtado y Cuenca López, 2017; Cuenca López et al., 2020).

A pesar de ello, sigue siendo necesaria la profundización en el estudio de las emociones en este campo de conocimiento, ya que probablemente es área de ciencias con menor presencia en el ámbito de las emociones.

Todo lo expuesto hasta ahora permite extraer dos ideas relevantes para el desarrollo de este trabajo:

- El estudio de las emociones en los procesos de enseñanza-aprendizaje ocupa un papel protagonista en las investigaciones educativas en la actualidad.

- El área de ciencias sociales es una de las áreas en las que menos se han desarrollado este tipo de análisis emocionales, y ofrece un interesante margen de aplicación para estudios de tipo emocional.

Dentro de los ámbitos en los que tienen una incidencia relevante las emociones, es importante destacar el valor de las pedagogías activas y emergentes, que con frecuencia se emplean para transformar los roles tradicionales del docente y del estudiante (Collazos, Guerrero, \& Vergara, 2001). Entre estas estrategias pedagógicas y metodológicas, la gamificación tiene una especial incidencia, tanto en la motivación, como en las emociones que se generan en docentes y estudiantes, en todas las etapas educativas y en todas las áreas de conocimiento (Segura-Robles, 2019). Algunos estudios señalan la importancia de esta metodología de gamificación en la disposición emocional de los estudiantes (Cejudo et al., 2019; Lavega et al., 2017; Mora \& Constain, 2016). El presente estudio se centra en el análisis de la incidencia de las emociones en procesos 
de enseñanza-aprendizaje en los que se han empleado metodologías de gamificación, que se caracterizan precisamente por su alto poder motivador en los estudiantes.

\section{Objetivos de la investigación}

Teniendo en cuenta las referencias que se han expuesto en la introducción, se ha diseñado una investigación sobre la incidencia de las emociones en los procesos de enseñanza-aprendizaje de las materias de ciencias sociales, en el ámbito de la Educación Secundaria, con experiencias didácticas diseñadas ad hoc, en las que se ha empleado la gamificación.

El objetivo principal de este estudio es analizar las emociones que se producen en los estudiantes de las asignaturas de ciencias sociales, en la etapa de Educación Secundaria, en intervenciones didácticas diseñadas desde la metodología de la gamificación.

Como objetivos secundarios, se plantean algunas cuestiones concretas:

- Valorar las emociones que se producen en los estudiantes de modo general durante la aplicación en el aula de estrategias de gamificación.

- Valorar la incidencia de la aplicación de esta estrategia metodológica en la visión que los estudiantes tienen de las materias de ciencias sociales

- Analizar posibles diferencias entre las emociones que se generan en relación con las diferentes estrategias de gamificación empleadas (juegos de narrativa, juegos digitales, juegos de mesa, escape room, breakout, juegos de rol y juegos de evaluación).

El planteamiento de estos objetivos parte de la base de que este tipo de metodologías activas fomenta la experimentación de diversos tipos de emociones en los estudiantes. En función de las emociones generadas en el proceso, se puede valorar la pertinencia del uso de la metodología diseñada para el área de conocimiento de las ciencias sociales.

\section{Material y método}

En este apartado se explican los parámetros en los que se ha llevado a cabo la aplicación de la estrategia pedagógica de gamificación en los diferentes cursos de la ESO, en las asignaturas pertenecientes al área de ciencias sociales.

\subsection{Estrategia pedagógica de gamificación y sus diversos tipos}

La estrategia didáctica que se ha empleado en esta experiencia sistemática es la metodología de la gamificación (Martínez et al., 2017).

Gamificar es aplicar estrategias (pensamientos y mecánicas) de juegos en contextos no jugables, ajenos a los juegos, con el fin de que las personas adopten ciertos comportamientos (Ramírez, 2014, p. 3).

Esta estrategia ha ido alcanzando protagonismo en los últimos años, generando experiencias de aplicación en todos los ámbitos de la enseñanza (Cortizo et al., 2011; Quintanal, 2016; Díez et al., 2017). También se pueden encontrar numerosas experiencias en el ámbito de las ciencias sociales, al que se ciñe este estudio (Carrión, 2018; Rivero, 2017). Algunas experiencias consisten en la aplicación de juegos como Minecraft para mejorare el desarrollo el pensamiento histórico (Guevara \& Colomer, 2017), o la aplicación de diversas experiencias para la adquisición de competencias históricas (Sánchez \& Colomer, 2018). Otras experiencias combinan la estrategia de gamificación y la aplicación de herramientas TIC para el desarrollo de competencias vinculadas con la historia (Gómez Trigueros, 2016) y con la geografía (Escamilla Ibáñez \& Moril Valle, 2017).

Entre las virtudes de esta metodología, destacan las siguientes:

- Es una metodología activa, que proporciona un alto grado de protagonismo de los estudiantes en el proceso de enseñanza-aprendizaje.

- Produce un alto nivel de motivación en los estudiantes, que afrontan el aprendizaje desde planteamientos psicológicos similares a los que se dan cuando se sitúan en escenarios de juego.

- Genera gran cantidad de emociones que, por regla general, mejoran la capacidad de aprendizaje de los estudiantes, aunque no siempre es así.

Estas características pedagógicas son las que se han tenido en cuenta para decantarse por esta estrategia didáctica en el diseño de la experiencia. 


\subsection{Participantes}

El grupo de participantes a los que se ha aplicado la experiencia es de un total de 280 estudiantes de diferentes centros de estudios, y de todos los cursos de Enseñanza Secundaria, en varios centros de Extremadura. La distribución de la muestra por cursos se presenta en la tabla 1.

Tabla 1

Distribución del grupo de participantes por cursos

\begin{tabular}{lll}
\hline Curso & $\begin{array}{l}\mathbf{N}^{\mathbf{o}} \text { de } \\
\text { estudiantes }\end{array}$ & Asignatura \\
\hline $1^{\circ}$ ESO & 62 & Geografía e Historia \\
$2^{\circ}$ ESO & 59 & Geografía e Historia \\
$3^{\circ}$ ESO & 55 & Geografía e Historia \\
$4^{\circ}$ ESO & 57 & Geografía e Historia y Economía \\
$1^{\circ}$ Bachillerato & 24 & Historia del mundo contemporáneo \\
$2^{\circ}$ Bachillerato & 23 & Historia del Arte \\
\hline
\end{tabular}

La distribución del grupo de participantes en función del género es equilibrada, aunque con mayor presencia de alumnas (56\%) que de alumnos (44\%).

En lo que se refiere a la distribución de los estudiantes de la muestra en función de la edad, es equiparable a la distribución por cursos académicos que se aprecia en la tabla 1. El grupo más numeroso pertenece a la etapa de Educación Secundaria Obligatoria, mientras que hay una representación minoritaria de estudiantes de Bachillerato. Esta distribución se puede apreciar en la figura 1.

\section{Figura 1}

Distribución de la muestra por edades

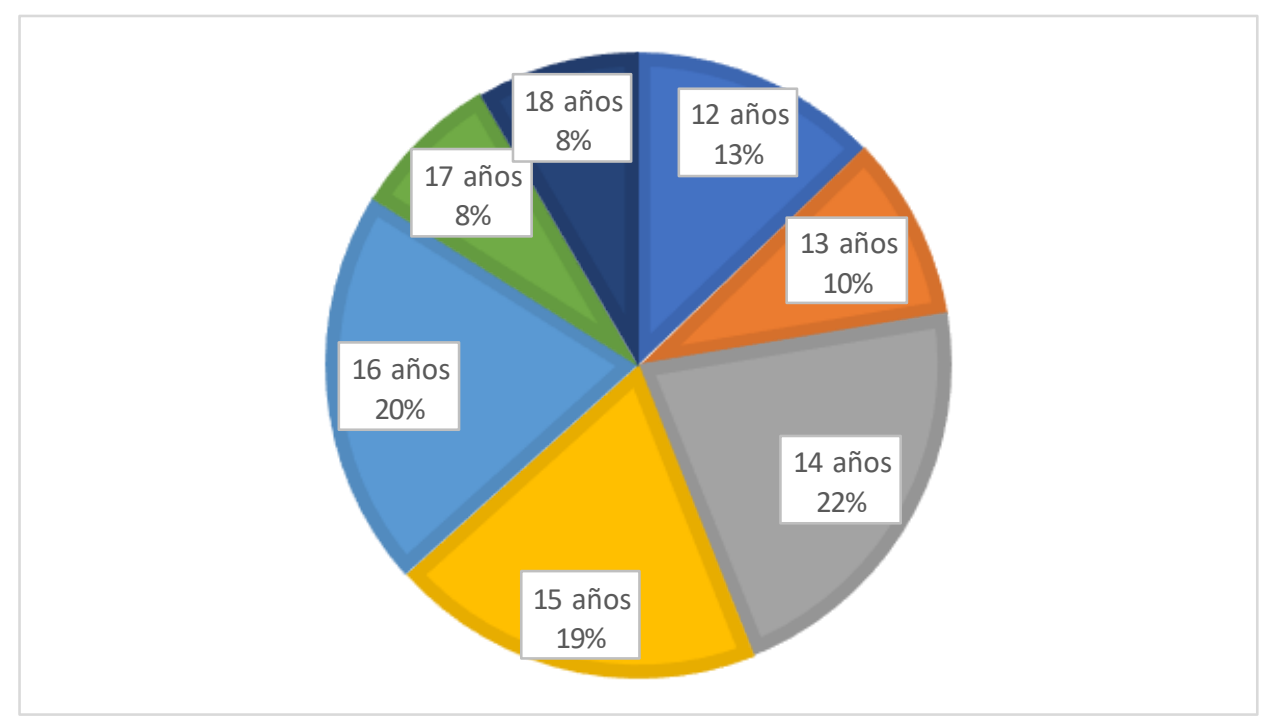

\subsection{Tipos de juegos utilizados en la experiencia}

Para la puesta en marcha de la experiencia, se han diseñado y aplicado una serie de actividades, en la que se combina el uso de estrategias de gamificación basadas en narrativa (Barreal \& Jannes, 2019), juegos digitales (Rodríguez et al., 2015), juego, experiencias de escape room (Sánchez-Martín et al. 2020; SeguraRobles \& Parra González, 2019); Breakout, juegos de rol y juegos de evaluación (Fuentes, 2019). En total, todos los grupos en los que se distribuye el grupo de participantes han tenido al menos ocho experiencias de gamificación a lo largo del curso 2019-2020. En total, se han aplicado 55 experiencias de gamificación sobre el conjunto de participantes. La distribución de estas experiencias se muestra en la tabla 2. 
Tabla 2

Distribución de las experiencias de gamificación en función de los tipos de juego y de los cursos en los que se ba aplicado

\begin{tabular}{|c|c|c|c|c|c|c|c|c|}
\hline Curso & $\begin{array}{l}\text { Juegos } \\
\text { de } \\
\text { narrativa }\end{array}$ & $\begin{array}{l}\text { Juegos } \\
\text { digitales }\end{array}$ & $\begin{array}{l}\text { Juegos } \\
\text { de } \\
\text { mesa }\end{array}$ & $\begin{array}{l}\text { Escape } \\
\text { Room }\end{array}$ & Breakout & $\begin{array}{l}\text { Juego } \\
\text { de rol }\end{array}$ & $\begin{array}{l}\text { Juego de } \\
\text { evaluación }\end{array}$ & $\begin{array}{l}\text { Sesiones } \\
\text { dedicadas }\end{array}$ \\
\hline $\begin{array}{l}1^{\circ} \\
\text { ESO }\end{array}$ & 1 & 1 & & & 2 & 2 & 3 & 16 \\
\hline & 1 & 2 & & 1 & 2 & 2 & 3 & 15 \\
\hline $\begin{array}{l}3^{\circ} \\
\mathrm{ESO}\end{array}$ & 1 & 2 & & 1 & 1 & 1 & 3 & 15 \\
\hline $\begin{array}{l}4^{\circ} \\
\mathrm{ESO}\end{array}$ & 1 & 1 & 1 & & & 2 & 3 & 14 \\
\hline $\begin{array}{l}1^{\circ} \\
\text { Bach }\end{array}$ & & 2 & 1 & & 2 & & 3 & 13 \\
\hline $\begin{array}{l}2^{\circ} \\
\text { Bach }\end{array}$ & & 2 & & 1 & 2 & 1 & 3 & 14 \\
\hline
\end{tabular}

El tiempo empleado en cada una de las actividades oscila entre una y tres sesiones de clase; las sesiones de juegos digitales se han podido llevar a cabo en una sesión de aula, mientras que los juegos vinculados a la narrativa, o los juegos de mesa, han necesitado dos o tres sesiones.

\subsection{Contenidos curriculares vinculados a las experiencias}

Uno de los requisitos que se ha tenido en cuenta en el diseño de la experiencia es la necesidad de que las actividades gamificadas estén vinculadas con los contenidos curriculares, para que los estudiantes puedan asociar la metodología aplicada con contenidos concretos de la materia que se está estudiando. La tabla 3 muestra la vinculación entre contenidos curriculares del currículo de educación Secundaria de Extremadura (Decreto 98/2016) y experiencias gamificadas en cada curso.

Tabla 3

Bloques curriculares en los que se ha empleado las actividades de gamificación (Textos tomados del Decreto 98/2016)

\begin{tabular}{|c|c|c|}
\hline Curso & Asignatura & Contenidos curriculares \\
\hline $1^{\circ} \mathrm{ESO}$ & Geografía e Historia & $\begin{array}{l}\text { a. Componentes básicos y formas de relieve. Componentes } \\
\text { básicos de las aguas. Tiempo atmosférico y clima. (p. 130) } \\
\text { b. El Mundo clásico, Roma: origen y etapas de la historia de } \\
\text { Roma; la república y el imperio: organización política y } \\
\text { expansión colonial por el Mediterráneo; el cristianismo (p. } \\
\text { 131). }\end{array}$ \\
\hline $2^{\circ} \mathrm{ESO}$ & Geografía e Historia & $\begin{array}{l}\text { La expansión comercial europea y la recuperación de las } \\
\text { ciudades. El arte románico, gótico e islámico (p. 136). }\end{array}$ \\
\hline $3^{\circ} \mathrm{ESO}$ & Geografía e Historia & $\begin{array}{l}\text { Actividades humanas: áreas productoras del } \\
\text { mundo. Sistemas y sectores económicos. } \\
\text { Espacios geográficos según actividad económica. } \\
\text { Los tres sectores Aprovechamiento y futuro de los recursos } \\
\text { naturales. Desarrollo sostenible. } \\
\text { Impacto medioambiental y aprovechamiento de } \\
\text { Recursos (p. 138) }\end{array}$ \\
\hline $4^{\circ} \mathrm{ESO}$ & Geografía e Historia & $\begin{array}{l}\text { El imperialismo en el siglo XIX: causas, desarrollo y } \\
\text { consecuencias. "La Gran Guerra". (1914-1919), o Primera } \\
\text { Guerra Mundial (p. 142). }\end{array}$ \\
\hline $4^{\circ} \mathrm{ESO}$ & Economía & $\begin{array}{l}\text { La idea de proyecto de empresa. } \\
\text { Evaluación de la idea. El entorno, el rol social de la empresa. } \\
\text { Elementos y estructura de la empresa. } \\
\text { El plan de empresa (p. 150) }\end{array}$ \\
\hline
\end{tabular}




\begin{tabular}{|c|c|c|}
\hline $1^{\circ}$ Bach. & $\begin{array}{l}\text { Historia del mundo } \\
\text { contemporáneo }\end{array}$ & $\begin{array}{l}\text { Revolución o revoluciones industriales: } \\
\text { características y causas. }\end{array}$ \\
\hline & & $\begin{array}{l}\text { Transformaciones técnicas y nuevas fuentes de energía. } \\
\text { Cambios debidos a la Revolución Industrial: transportes, } \\
\text { agricultura, población (migraciones } \\
\text { y nuevo concepto de ciudad). }\end{array}$ \\
\hline $2^{\circ} \mathrm{Bach}$ & Historia del Arte & $\begin{array}{l}\text { a. Grecia, creadora del lenguaje clásico. Principales } \\
\text { Manifestaciones (p. 779). } \\
\text { b. El Renacimiento. Mecenas y artistas. Origen y } \\
\text { desarrollo del nuevo lenguaje en arquitectura, } \\
\text { escultura y pintura. Aportaciones de los grandes artistas del } \\
\text { Renacimiento italiano (p. 784). }\end{array}$ \\
\hline
\end{tabular}

\subsection{Análisis de resultados}

Para analizar los resultados de la experiencia, se ha llevado a cabo un estudio de los diarios de aprendizaje de los grupos de estudiantes, a través de una metodología cualitativa (Álvarez-Gayou, 2003; Souza, et al. 2016). La herramienta del diario de aprendizaje resulta de una gran utilidad para observar cómo viven los estudiantes los procesos de aprendizaje. Por este motivo, se ha considerado idónea para el fin que persigue esta investigación.

La elección de esta metodología de análisis se debe al interés que suscita para este estudio la posibilidad de explorar la narrativa de las emociones que los propios estudiantes hacen después de haber trabajado unos contenidos didácticos desde una metodología como la gamificación. En contraposición con la información que nos podría ofrecer un análisis de tipo cuantitativo, que mostraría una foto fija, este método de análisis permite conocer el modo concreto en que cada participante ha vivido la experiencia que se analiza. En este sentido, se describe de este modo la investigación cualitativa:

Es una actividad sistemática orientada a la comprensión en profundidad de fenómenos educativos y sociales, a la transformación de prácticas y escenarios educativos, a la toma de decisiones y al descubrimiento de un cuerpo organizado de conocimientos. (Sandín Esteban, 2003, p. 123).

Esta metodología de análisis viene avalada en su fiabilidad por diversos estudios contrastados (ÁlvarezGayou, 2003; Bisquerra Alzina, 2004; Kornblit, 2007), que permiten utilizarla como un medio fiable para la obtención de resultados de investigación en estudios educativos.

Para la redacción de los diarios de aprendizaje, se ha pedido a los estudiantes que redacten una entrada en su diario al finalizar cada experiencia de gamificación. Las instrucciones proporcionadas para la redacción de estas entradas son las siguientes:

- De debe elaborar un texto en el que se responda de modo narrativo (como un único texto, y no como respuestas a las preguntas) ¿qué contenidos ha aprendido? ¿cómo ha sido la actividad a través de la cual lo ha aprendido? Y ¿cómo se ha sentido durante la realización de la actividad? ¿ha cambiado en algo tu manera de ver la asignatura después de esta actividad?

- Además, se indica a los estudiantes que los textos han de se elaborados en un documento digital, y deben contener entre 150 y 200 palabras.

\section{Resultados y discusión}

Para el análisis de los textos seleccionados en los diarios de los estudiantes participantes en la muestra, se ha realizado un proyecto en WebQDA, estableciendo una red sencilla de categorías, que se corresponde con las emociones básicas que inciden en el proceso de enseñanza-aprendizaje (Lavega et al., 2017). La figura 2 muestra de modo esquemático esta red de categorías. 


\section{Figura 2}

Red de categorías con las que se han estudiado los textos de los diarios de los estudiantes (basado en Lavega, et al., 2017).

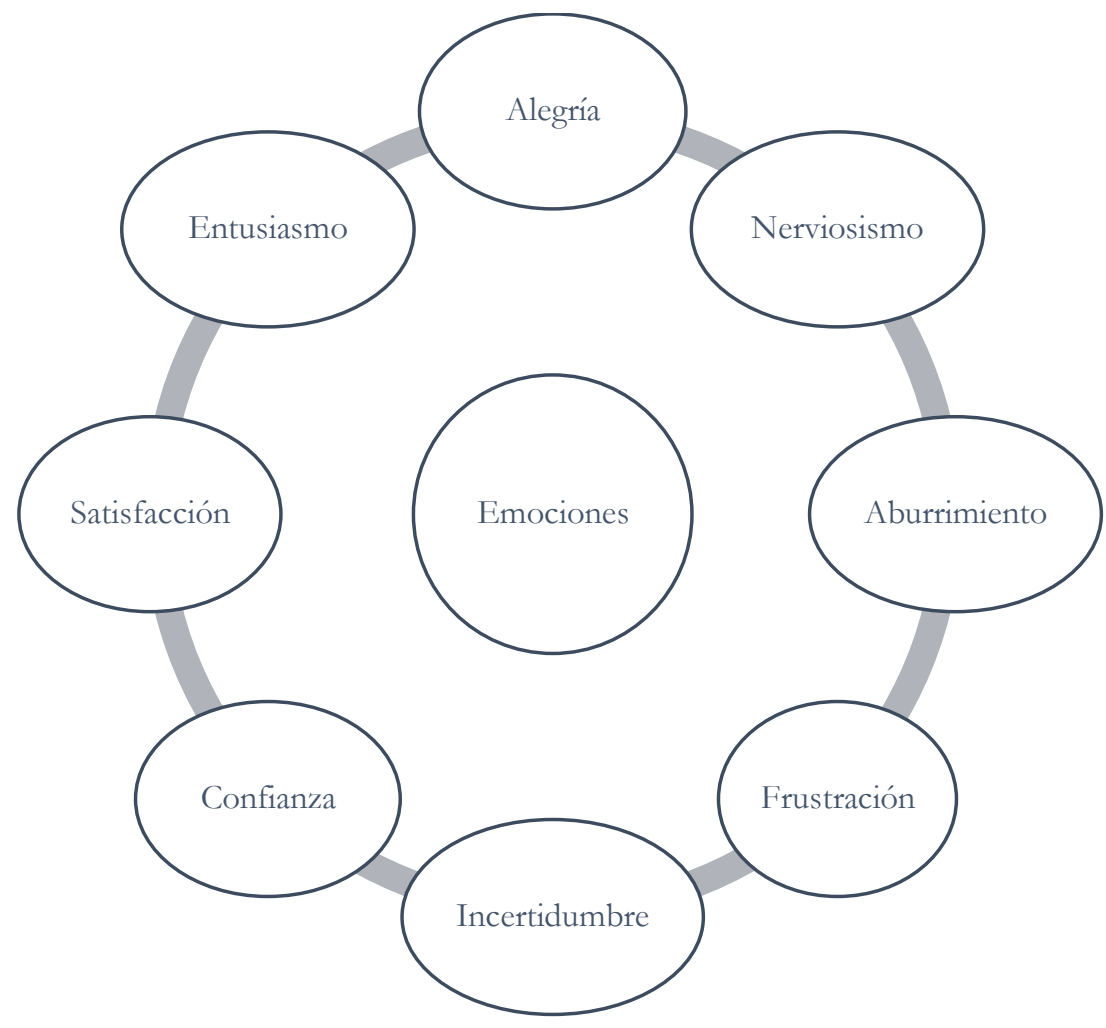

Una vez diseñada la red, se han introducido los textos en el programa, y se han asociado a cada categoría los fragmentos en los que se alude directa o indirectamente a cada una de las emociones. Este proceso ha permitido obtener los resultados que se explican a continuación.

En primer lugar, se ha hecho un análisis de las emociones identificadas y categorizadas en los textos según la categorización de emociones que se muestran en la figura anterior. Se han categorizado en total 378 fragmentos en los que se localizan expresiones compatibles con alguna de las emociones analizadas. La distribución de estas emociones muestra claramente una mayor incidencia de unas emociones que de otras. Emociones como la alegría o el entusiasmo, predominan sobre las demás, aunque también hay una gran presencia de la incertidumbre, y en algunos casos, de la frustración. La figura 3 muestra la relación entre las emociones y la cantidad de fragmentos que se han categorizado. 
Figura 3

Frecuencia de textos categorizados en relación con cada emoción

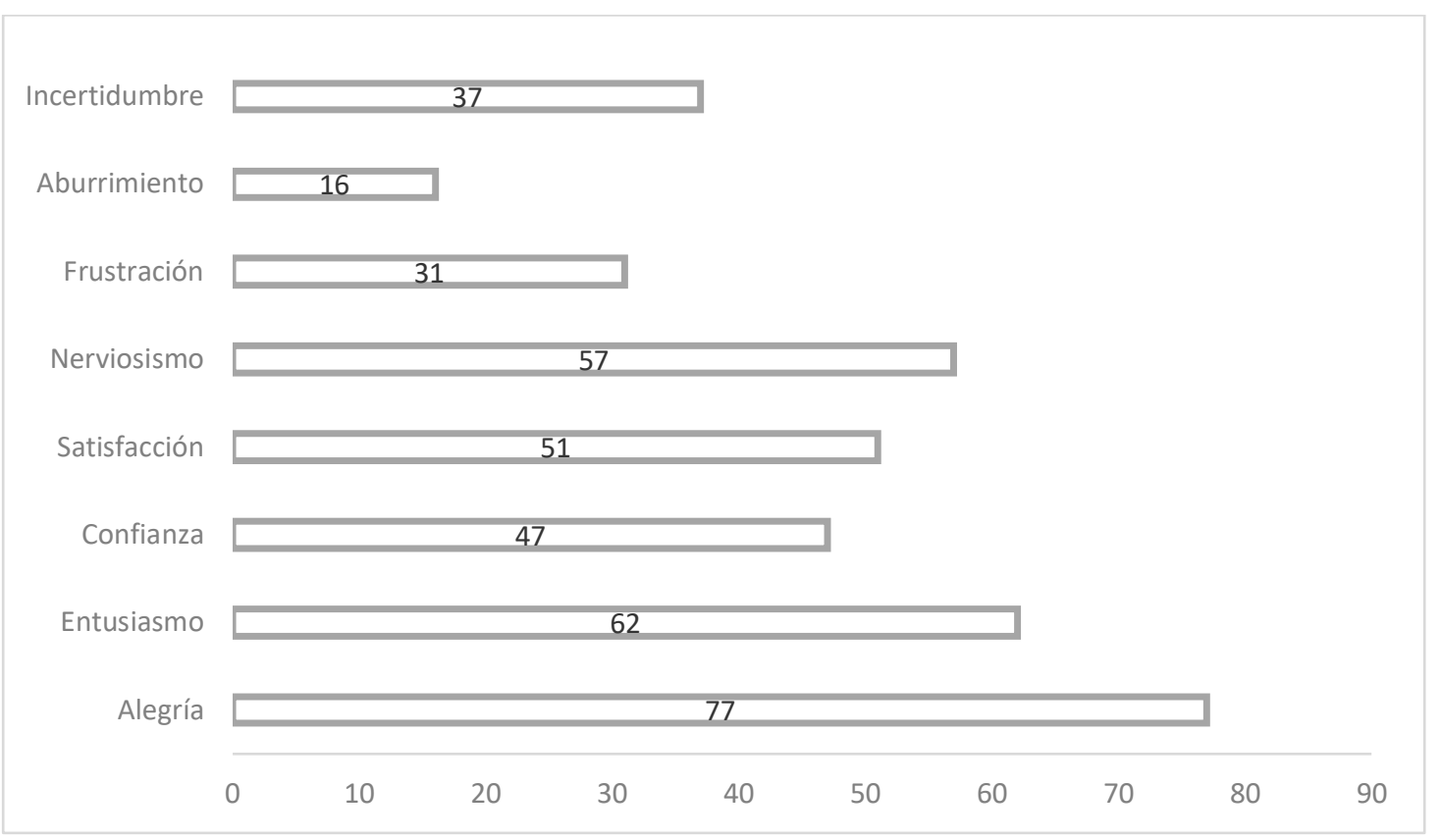

Si se agrupa el número de fragmentos de texto categorizados en función de cómo puede influir en el aprendizaje de los estudiantes, las emociones que influyen de modo positivo, 237 en total (alegría, entusiasmo, satisfacción o confianza), predominan sobre las que inciden de modo negativo, 141 en total (nerviosismo, aburrimiento, frustración, o incertidumbre).

Esta categorización se puede observar en algunos fragmentos de redacción de los diarios de aprendizaje de algunos estudiantes, que se muestra a continuación a modo de ejemplo, para hacer más comprensible el proceso de categorización que se ha aplicado:

Sujeto de $2^{\circ}$ de ESO manifiesta satisfacción tras la realización de un juego digital en la asignatura de Geografía e Historia:

Esta semana hemos hecho un juego de preguntas y respuestas a través de una app. Me ha gustado mucho ya que ha sido una actividad muy divertida y que te ayuda a trabajar en grupo. También me ha ayudado a recordar las cosas que hemos aprendido en este tema.

Sujeto de $1^{\circ}$ de Bachillerato muestra entusiasmo tras la realización de una actividad Breakout:

La actividad de Breakout que hemos hecho esta semana, sobre los tipos de sociedad me ha encantado, porque es un juego, pero a la vez, sirve para recordar las cosas que hemos aprendido en este tema. Estudiar así es mucho mejor que aprenderlo del libro.

Sujeto de $1^{\circ}$ de ESO manifiesta alegría al resolver un juego de evaluación sobre el antiguo Egipto:

Me ha gustado mucho la actividad del juego digital. Estoy muy contenta de haber resuelto el juego, porque eso quiere decir que me he aprendido bien el tema. La verdad es que estos juegos aparte de que están muy bien hechos y preparados es una forma muy buena de que se te queden las cosas y aprendértelas. Así que para mí un 10/10.

Sujeto de $4^{\circ}$ de ESO manifiesta entusiasmo tras realizar un juego digital:

El juego sobre los sectores económicos me ha gustado bastante, diría yo. Ha sido una forma muy dinámica y atrayente de que los alumnos/as se hayan entretenido (hasta cierto punto) con el contenido de la asignatura. Espero que en otros temas hagamos más.

Varios sujetos manifiestan combinaciones de emociones como nerviosismo, incertidumbre, satisfacción o alegría tras realizar una actividad escape room:

Al principio estaba muy intrigado por la música y al final me ha gustado mucho por el misterio.

Al principio bien pero conforme iba pasando el tiempo me ponía más nerviosa, pero me ha gustado mucho me gustan mucho las cosas de misterio.

Me he sentido, a veces, un poco atascada, pero me ha gustado muchísimo.

Durante la actividad ha habido momentos en los que me lo he pasado bien y otros en los que me sentía aburrido porque había demasiadas ecuaciones para hacer. 
Para responder a la cuestión planteada por el segundo de los objetivos secundarios de la investigación, se han analizado fragmentos en los que los estudiantes hacen referencia a su visión acerca de las ciencias sociales. Especialmente, se han tenido en cuenta aquellos textos en los que los estudiantes responden a una cuestión que se le solicitaba específicamente en el diario de aprendizaje. Esta pregunta es cha cambiado en algo tu manera de ver la asignatura después de esta actividad? Los fragmentos en los que los estudiantes responden a esa cuestión se han categorizado en función de tres variables posibles: "mejora la visión de la asignatura", "empeora la visión de la asignatura" o "se mantiene la visión de la asignatura". A través de esta categorización se detecta un considerable número de estudiantes que se expresan de modo compatible con la categoría "mejora la visión de la asignatura" como se presenta en la distribución en los textos de la figura 4.

\section{Figura 4}

Datos de categorización de los textos sobre la visión de los estudiantes, en relación con las tres categorias (la visión de los estudiantes sobre la asignatura "se mantiene", "empeora", "mejora")

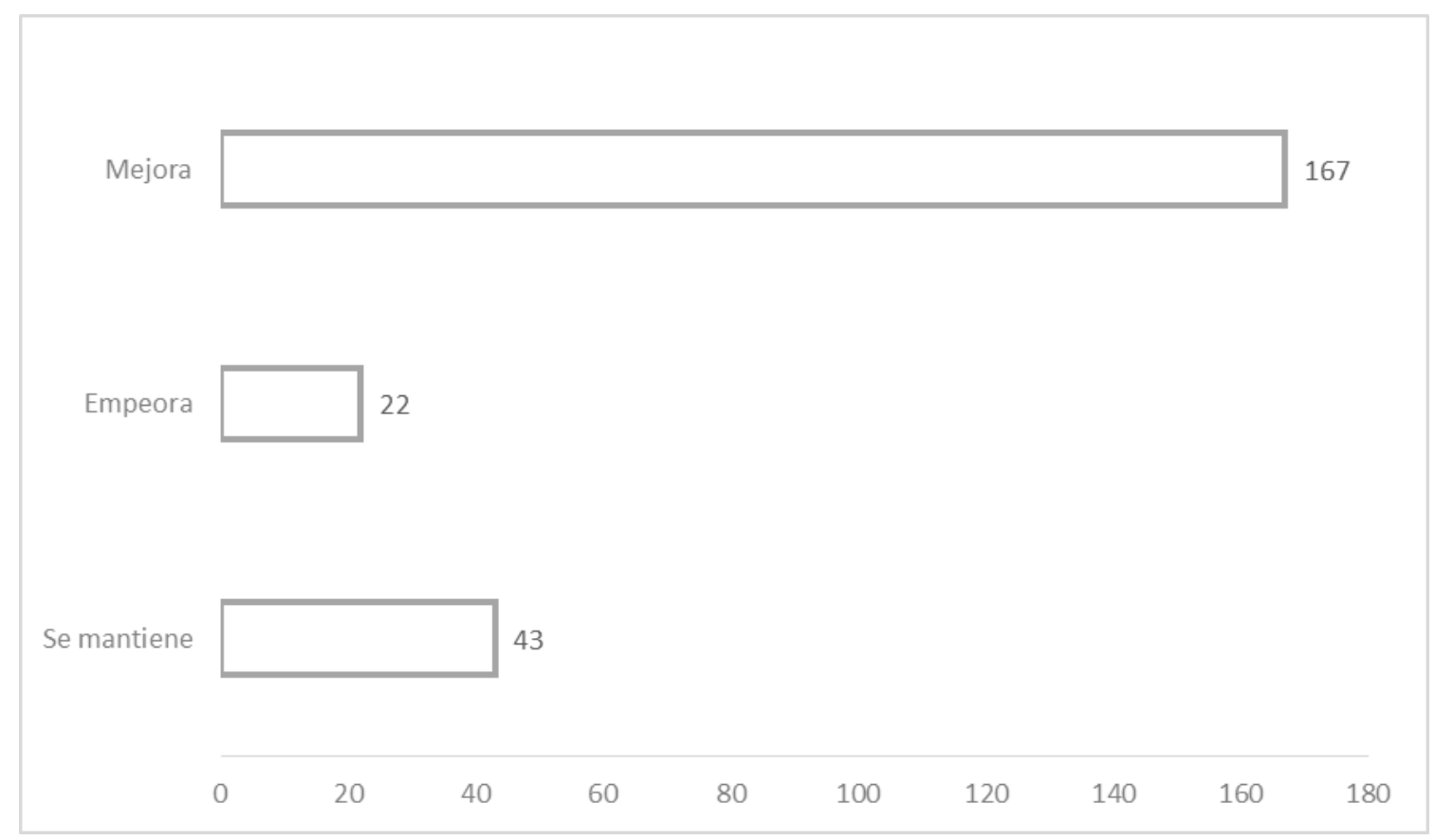

Los datos de la figura se complementan con algunos ejemplos concretos, extraídos de los diarios de aprendizaje, que se presentan a continuación:

Sujeto de $2^{\circ}$ de bachillerato muestra una mejor visión de la materia tras realizar juegos de tipo breakout en la asignatura de historia del arte:

Con estos juegos te tomas la asignatura de otra manera. Aunque hay mucho que estudiar, muchos nombres de obras y artistas, muchas épocas, el juego que ayuda a recordar mejor cada cosa. No pensaba que en una asignatura de $2^{\circ}$ de Bachillerato se pudieran usar juegos para aprender.

Sujeto de 1 de ESO mejor a su visión de la asignatura después de un juego de narrativa sobre Objetivos de Desarrollo Sostenible aplicado a los contenidos de geografía durante todo el curso:

A mí me gustan un montón las misiones, porque me gusta mucho hacer trabajos y presentaciones, me interesa lo de los ODS, y no me importa trabajarlo. Esta asignatura ha sido como un juego en el que nos hemos convertido en superhéroes. No recordaba que me gustase tanto en otros cursos.

Sujeto de $3^{\circ}$ de ESO manifiesta la misma visión de la asignatura tras una actividad de juego de rol en la asignatura de geografía:

Me gusta esta asignatura porque de vez en cuando nos toca hacer algún juego que hace la clase más amena, y no es sólo estudiar y memorizar.

Sujeto de $3^{\circ}$ de ESO que manifiesta una visión negativa de la asignatura de geografía e historia tras fracasar intentando hacer un Breakout:

Esta semana hemos hecho un juego que no he sido capaz de resolver, porque cada vez que no sabía algo me mandaba empezar desde el principio. Ya me ha pasado otras veces en la asignatura, y no sé que hacer para que me salga mejor. 
En lo que se refiere a la incidencia de emociones en las actividades, según las diferentes estrategias de gamificación empleadas, se aprecia una vinculación entre tipos de emociones que se generan en algunos tipos de juego. Por ejemplo, es habitual que en actividades de escape room haya estudiantes que manifiestan a la vez, entusiasmo y nerviosismo. También se detecta en ocasiones, frustración en los juegos de evaluación, si no se logran los objetivos. La figura 5 muestra la incidencia de emociones por modalidades de juegos.

\section{Figura 5}

Distribución de las emociones categorizadas en función de las actividades de gamificación

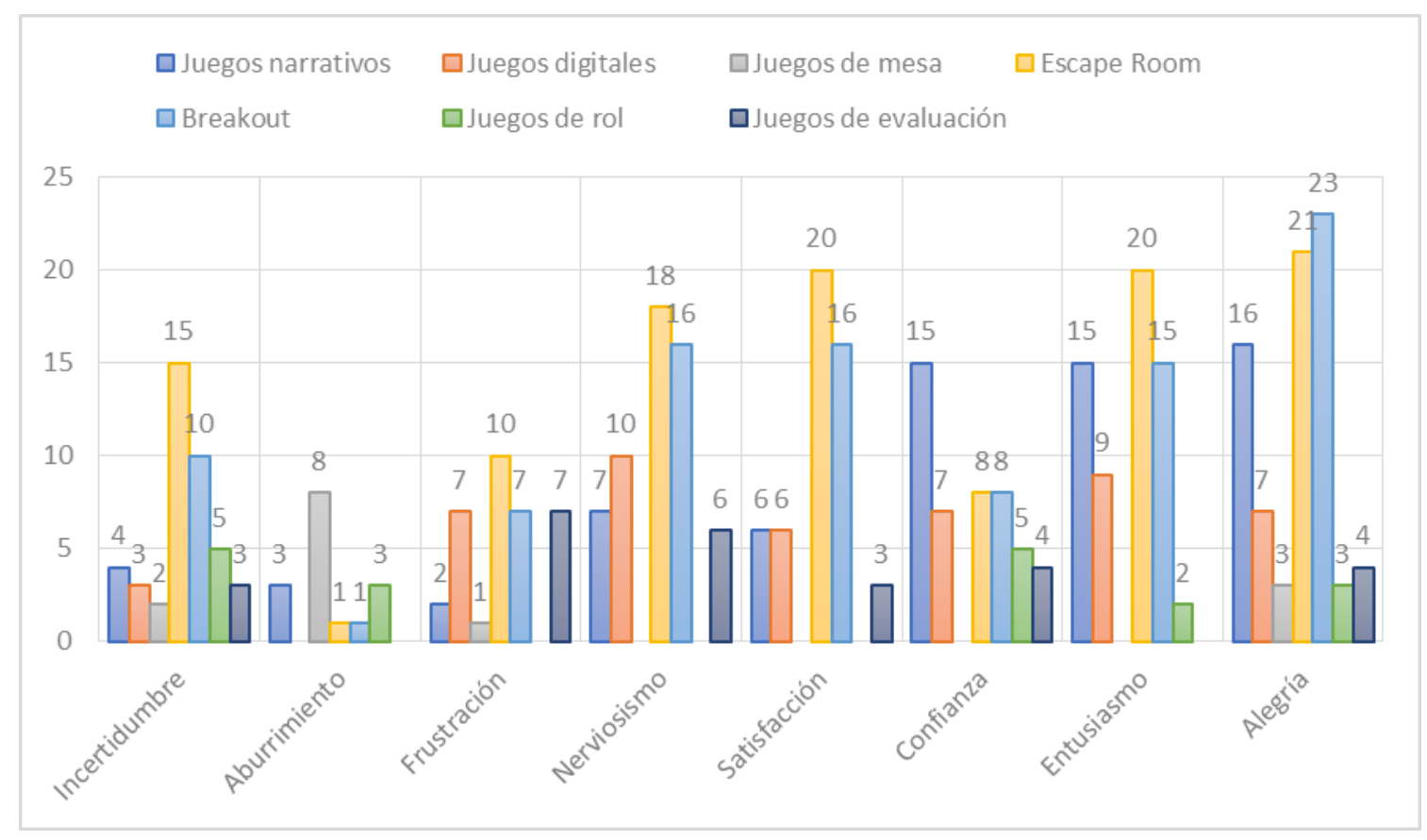

Los resultados obtenidos son coherentes con los que reportan otros estudios, como el de Lavega et al. (2017), que informa acerca de cómo los juegos reportan una gran cantidad de emociones a los estudiantes, tales como la alegría o el entusiasmo, y sirven para incrementar la motivación. Por su parte, Teixes (2015), en su análisis de la gamificación, elabora un estudio de interés acerca de las causas por las que esta metodología genera emociones; en este sentido, destaca el papel de la motivación que generan estas actividades en los estudiantes. A pesar de estos beneficios, algunos estudios señalan los aspectos negativos, como las tensiones o el aburrimiento, que a vece puede generarse (Carrión, 2018).

La visión positiva acerca de las asignaturas, que se observa mayoritariamente en los textos analizados, como consecuencia de la aplicación de estas estrategias de gamificación es señalada también en otros estudios. Carrión (2018) llama la atención acerca del poder motivador de los juegos en las materias de ciencias sociales; Gómez Muñiz (2020) ha llevado a cabo un estudio acerca de los efectos de la aplicación de juegos a través de realidad aumentada en materias de esta misma área, destacando también el alto grado de motivación. Dada la habitual falta de motivación de los estudiantes en estas materias (Corrales, et al., 2018; 2020), resulta relevante constatar el grado de motivación y las emociones positivas de cara al aprendizaje que se genera con estas estrategias didácticas.

Por último, estudios realizados sobre los diferentes tipos de juegos, refieren, por ejemplo, la disparidad de emociones que se generan en escape room o Breakout (Moreno, 2018; García-Lázaro, 2019) o el interés que suscitan los juegos digitales (Lavega et al., 2017).

\section{Conclusiones}

A la vista de los resultados que se han obtenido en las pruebas aplicadas, se pueden extraer una serie de conclusiones de interés para la mejora de los procesos de enseñanza-aprendizaje de las ciencias sociales.

En función del objetivo principal que se plateaba en esta investigación, analizar las emociones que se producen en los estudiantes de las asignaturas de ciencias sociales, en la etapa de Educación Secundaria, en intervenciones didácticas diseñadas desde la metodología de la gamificación. Por un lado, se verifica la utilidad y el interés del conocimiento de las emociones que se generan en los estudiantes en el aprendizaje 
de las materias vinculadas a las ciencias sociales. A pesar de que no son muchos los estudios que analizan las emociones en esta disciplina, se ha podido ver que el conocimiento de las emociones que se generan permite implementar mejoras en el aprendizaje de los estudiantes.

En relación con el primero de los objetivos secundarios, Valorar las emociones que se producen en los estudiantes durante la aplicación en el aula de estrategias diferentes de gamificación, se aprecia una incidencia alta de emociones, como la alegría o el entusiasmo, que inciden positivamente en el aprendizaje, aunque también se abrevia aburrimiento, y en ocasiones, nerviosismo o agobio, en las experiencias llevadas a cabo con estrategia metodológica de gamificación. Tanto el análisis de las experiencias, como en la discusión con otros trabajos que analizan el mismo tipo de experiencias, permiten justificar que la gamificación genera en los estudiantes una serie de emociones, que son reconocibles por los propios estudiantes, y que repercuten positivamente en el aprendizaje de los contenidos. Así lo manifiestan en los textos analizados de los diarios de aprendizaje de los estudiantes. Esta circunstancia permite aconsejar la implementación de esta metodología en el aprendizaje de las ciencias sociales.

A pesar de los beneficios que parecen ponerse de relieve en el análisis de las experiencias que se ha llevado a cabo, también se puede constatar el hecho de que, en algunos casos concretos, los estudiantes manifiestan emociones negativas, como la frustración por no conseguir los objetivos que la actividad plantea, o el estrés durante el desarrollo de algunos de los juegos en los que el tiempo o los propios compañeros presionan al resto. Este elemento ha de ser tenido en cuenta a la hora de implementar actividades de gamificación, para tratar de reducir en la medida de lo posible estas emociones negativas.

Otro aspecto que se debe manejar con las precauciones adecuadas es la competitividad que, en ocasiones, viene de la mano de estos procesos de gamificación. Por un lado, es interesante que los estudiantes se motiven en función de la consecución de los objetivos, pero, por otro lado, hay que tener en cuenta que un alto grado de competitividad genera emociones negativas que se han expuesto anteriormente y, además, puede tener influencias negativas en la dinámica de la clase.

En relación con la visión que los estudiantes quieren acerca de las materias de ciencias sociales, aludida en el segundo objetivo, se observa que esta estrategia didáctica incide positivamente en la visión que los estudiantes tienen de las asignaturas, mejorando en un amplio número de sujetos de la muestra.

En lo que se refiere al análisis de las posibles diferencias entre las emociones que se generan en función de las diferentes estrategias de gamificación empleadas (tercer objetivo secundario)., se observa que hay algunos tipos de juego, como los de mesa o los narrativos, que tienen una incidencia media de emociones, ya que se registra un número moderado de emociones, y casi siempre de signo positivo. Sin embargo, algunos juegos vinculados con herramientas digitales, o juegos de alta intensidad, como escape room o Breakout generan incidencias altas de emociones positivas y negativas. Además, los juegos basados en la competitividad generan en ocasiones emociones negativas, como la frustración, además de un exceso de competitividad.

Para mitigar estas posibles consecuencias, se recomienda tener en cuenta este factor en el diseño de la actividad. Modalidades de gamificación, como el escape room o el Breakout son más propensas a generar estas situaciones de agobio y estrés, aunque también producen mucha motivación. Otras actividades como los juegos digitales y de mesa permiten diseñar actividades menos afectadas por este tipo de circunstancias negativas, pero pueden hacerse más monótonas para los estudiantes.

La valoración de todas estas circunstancias permite concluir que la gamificación es una metodología que genera emociones que repercuten positivamente en el proceso de enseñanza-aprendizaje de las materias de ciencias sociales, analizadas en el presente estudio.

\section{Referencias}

Aguaded Gómez, M. y Pantoja Chaves, M. J. (2015). Innovar desde un proyecto educativo de inteligencia emocional en primaria e infantil. Tendencias pedagogicas, 26, 68-88. http://hdl.handle.net/10486/668043

Álvarez-Gayou, J.L. (2003). Cómo hacer investigación cualitativa. Fundamentos y metodología. Paidós.

Barreal, J. y Jannes, G. (2019). La narrativa como herramienta docente dentro de la gamificación de la estadística en el Grado en Turismo. Digital Education Review, 36, 152-170.

Bisquerra Alzina, R. (2005). La educación emocional en la formación del profesorado. Revista interuniversitaria de formación del profesorado, 19(3), 95-114.

Bisquerra Alzina, R. (2004). Metodología de la investigación educativa. Editorial La Muralla.

Bisquerra Alzina, R. y Hernández Paniello, S. (2017). Psicología positiva, educación emocional y el programa aulas felices. Papeles del Psicólogo, 38(1), 58-65. https://doi.org/10.23923/pap.psicol2017.2822

Brígido, M., Caballero, A., Bermejo, M. L. y Mellado, V. (2009). Las emociones sobre la enseñanza y aprendizaje de las ciencias en estudiantes de maestros de primaria. Revista electrónica de motivación y emoción, 
$11(31)$ 514-532.

Bolaños Florido, L. P. (2016). El estudio socio-histórico de las emociones y los sentimientos en las Ciencias Sociales del siglo XX. Revista de Estudios Sociales, 55, 178-191.

Borrachero, A. B. (2015). Las emociones en la enseñanza y el aprendizaje de las ciencias en educación secundaria. Enseñanza de las Ciencias: revista de investigación y experiencias didácticas, 33(3), 199-200.

Broc Cavero, M. Á. (2019). Inteligencia emocional y rendimiento académico en alumnos de educación secundaria obligatoria. Revista española de orientación y psicopedagogía, 30(1), 75-92 DOI: https://doi.org/10.5944/reop.vol.30.num.1.2019.25195

Carrión, E. C. (2018). El uso de la gamificación y los recursos digitales en el aprendizaje de las ciencias sociales en la educación superior. DIM: Didáctica, Innovación y Multimedia, 36, 1-11.

Cera, E., Bartolomé, J., Conde, C. y Sáez, P. (2015). Inteligencia emocional y motivación en educación física en secundaria. RETOS. Nuevas Tendencias en Educación Física, Deporte y Recreación, 27, 8-13.

Cejudo, J., Losada, L., Garrido, M. P. y Feltrero, R. (2019). Programa "aislados": la gamificación como estrategia para promover el aprendizaje social y emocional. Voces de la Educación, no especial 2, 155-168.

Collazos, C., Guerrero, L. y Vergara, A. (2001). Aprendizaje Colaborativo: un cambio en el rol del profesor. En Proceedings of the 3 rd Workeshop on Education on Computing. Punta Arenas.

Corrales, M., Díaz, B., Sánchez, J. y Moreno, J. (2019). El laboratorio de humanidades y ciencias sociales en educación secundaria. Papeles salmantinos de educación, 23, 129-151.

Corrales, M., Sánchez, J., Moreno, J. y Zamora, F. (2018). Las motivaciones de los jóvenes para el estudio: raíces y consecuencias. Cuadernos de Investigación en Juventud, 4, 60-79. doi: 10.22400/cij.4.e020

Corrales, M., Moreno, J., Sánchez J. y Polo, F. Z. (2020). Estudio cualitativo de las motivaciones del alumnado de bachillerato en referencia a la modalidad de estudios. Campo Abierto. Revista de Educación, 39(1), 85-99. DOI: 10.17398/0213-9529.39.1.85.

Cortizo, J. C., Carrero, F., Monsalve, B., Velasco, A., Díaz del Dedo, L. I. y Pérez Martín, J. (2011). Gamificación y Docencia: Lo que la Universidad tiene que aprender de los Videojuegos. En M. J. García García y E. Icarán (Coords.), VIII Jornadas internacionales de innovación universitaria. Universidad Europea de Madrid.

Cuenca-López, J. M. y Estepa-Giménez, J. (2017). Educación patrimonial para la inteligencia territorial y emocional de la ciudadanía. MID AS. Museus e estudos interdisciplinares, 8.

Cuenca-López, J. M., Martín-Cáceres, M. J. y Estepa-Giménez, J. (2020). Buenas prácticas en educación patrimonial: análisis de las conexiones entre emociones, territorio y ciudadanía. Aula abierta, 49(1), 4554.

Díez, J. C., Bañeres, D. y Serra, M. (2017). Experiencia de gamificación en Secundaria en el Aprendizaje de Sistemas Digitales. Education in the Knowledge Society, 18(2), 85-105.

Domínguez, J., Domínguez, V., López, M. E. y Rodríguez, M. del M. (2016). Motivación e inteligencia emocional en estudiantes de Educación Secundaria Obligatoria. Revista de estudios e investigación en psicología y educación, 3(2), 94-101. https://doi.org/10.17979/reipe.2016.3.2.1801

Escamilla Ibáñez, J. D. y Moril Valle, R. (2017). El aprendizaje de la Geografía a través de la gamificación. Trópico, 4, 122-128.

Fernández-Berrocal, P., Cabello-González, R. C. y Gutiérrez-Cobo, M. J. G. (2017). Avances en la investigación sobre competencias emocionales en educación. Revista interuniversitaria de formación del profesorado, 88, 15-26.

García-Lázaro, I. (2019). Escape Room como propuesta de gamificación en educación. Hekademos: revista educativa digital, 27, 71-79.

Gómez-Hurtado, I. y Cuenca López, J. M. (2017). Trabajar las emociones desde la educación patrimonial para atender a la diversidad del aula. En R. Martínez Medina, R. García-Morís y C. R. García Ruíz (Eds.), Investigación en didáctica de las ciencias sociales. Retos preguntas y lineas de investigación (pp. 649-657). Universidad de Córdoba.

Gómez Muñiz, V. (2020). Merge Cube, la realidad aumentada al servicio de las ciencias sociales. Iber: Didáctica de las ciencias sociales, geografía e historia, 98, 80-82.

Gómez Trigueros, I.M. (2016) La didáctica de la Geografía y las TIC: nuevas metodologías de enseñanza y aprendizaje. En J.D. Álvarez, S. Grau y M.T. Tortosa (Coords.), Innovaciones metodológicas en docencia universitaria: resultados de investigación (pp. 839-849). Universidad de Alicante.

Guevara, J. M. y Colomer, J. C. (2017). Minecraft y Eduloc, en historia y geografía. Iber: Didáctica de las ciencias sociales, geografía e historia, 86, 16-23.

Hargreaves, A. (2003). Teaching in the knowledge society: Education in the age of insecurity. Teachers College Press.

Kelchtermans, G. \& Deketelaere, A. (2016). The emotional dimension in becoming a teacher. In J. Loughran \& M. L. Hamilton (Eds.) International handbook of teacher education (pp. 429-461). Springer. 
Kornblit, A. L. (2007). Metodologías cualitativas en ciencias sociales: modelos y procedimientos de análisis. Biblos.

Lavega, P., Filella, G., Agulló, M. J., Soldevila, A. y March, J. (2017). Conocer las emociones a través de juegos: Ayuda para los futuros docentes en la toma de decisiones. Electronic Journal of Research in Education Psychology, 9(24), 617-640.

López Cassà, È. L. (2016). La educación emocional en la Educación Infantil y Primaria. En J. L. Soler, L. Aparicio, O. Díaz, E. Escolano, y A. Rodríguez, A. (Eds.), Inteligencia Emocional y Bienestar II: reflexiones, experiencias profesionales e investigaciones (pp. 557-570). Ediciones Universidad de San Jorge.

Mellado, V., Borrachero, A. B., Brígido, M., Melo, L. y Dávila, M. A. (2014). Las emociones en la enseñanza de las ciencias. Enseñanza de las ciencias: revista de investigación y experiencias didácticas, 32(3), 11-36.

Martínez, A., Rodríguez, K., Ochomogo, Y. y Miguelena, R. (2017). Gamificación: La enseñanza divertida. El Tecnológico, 28(1), 9-11.

Miralles Pascual, R., Filella Guiu, G. y Lavega i Burgués, P. (2017). Educación física emocional a través del juego en educación primaria: ayudando a los maestros a tomar decisiones. Retos: Nuevas tendencias en Educación Física, Deporte y Recreación, 31, 88-93. http://hdl.handle.net/10459.1/59188

Mora, P. A. y Consatain, G. (2016). La Gamificación como recurso tecnológico para el desarrollo de Inteligencia Emocional. En VII Coloquio Internacional de Educación.

Moreno, E. (2019). El "Breakout EDU" como herramienta clave para la gamificación en la formación inicial de maestros/as. Edutec. Revista Electrónica De Tecnología Educativa, 67, 66-79. https://doi.org/10.21556/edutec.2018.66.1247

Quintanal, F. (2016). Gamificación y la Física-Química de Secundaria. Education in the Knowledge Society, 17(3), 13-28. http://hdl.handle.net/10366/ 132127.

Ramírez, J.L. (2014). Gamificación. Mecánicas de juegos en tu vida personaly profesional. Servicio Comercial del Libro.

Retana Alvarado, D. A., De las Heras Pérez, M. Á., Jiménez Pérez, R. y Vázquez Bernal, B. (2017). Emociones de maestros en formación inicial sobre la Didáctica de las Ciencias antes de una intervención indagatoria. Enseñanza de las Ciencias, número extraordinario, 5415-5422.

Rivero, M. P. (2017). Procesos de gamificación en el aula de ciencias sociales. Iber: Didáctica de las ciencias sociales, geografia e historia, 86, 4-6.

Rodríguez, F., Loro, F. y Villén, S. (2015). Experiencia de gamificación en alumnos de magisterio para la evaluación de la asignatura Sociología de la educación mediante el uso de la plataforma Kahoot. En 3rd International conference on innovation, documentation and teaching technologies (p. 223).

Sánchez-Álvarez, N., Extremera, N. \& Fernández-Berrocal, P. (2015). Maintaining life satisfaction in adolescence: Affective mediators of the influence of perceived emotional intelligence on overall life satisfaction judgments in a two-year longitudinal study. Frontiers in Psychology, 6, 1892.

Sánchez-Martín, J., Corrales-Serrano, M., Luque-Sendra, A. y Zamora-Polo, F. (2020). Exit for success. Gamifying science and technology for university students using escape-room. A preliminary approach. Heliyon, 6(7), e04340. DOI: 10.1016/j.heliyon.2020.e04340

Sánchez Calleja, L., Rodríguez Gómez, G. y García Jiménez, E. (2018). Desarrollo de competencias emocionales a través del Programa AEdEm para Educación Secundaria. Universidad de Sevilla.

Sánchez, Á. y Colomer, J. C. (2018). Gamificación y construcción del pensamiento histórico: desarrollo de competencias en actividades gamificadas. Clio: History and History Teaching, 44, 82-93.

Sandín Esteban, M. P. (2003). Investigación cualitativa en educación. Fundamentos y tradiciones. Mc Graw and Hill Interamericana.

Segura-Robles, A. (2019). Producción científica sobre gamificación en educación: un análisis cienciométrico Scientific production about gamification in education: A Scientometric analysis. Revista de Educación, 386, 113-135.

Segura-Robles, A. \& Parra González, M. E. (2019). How to implement active methodologies in Physical Education: Escape Room. ESHPA Educ. Sport Health Phys, act. 3, 295-306.

Souza, F. N. de, Costa, A. P., Moreira, A., Souza, D. N. de y Freitas, F. (2016). WebQDA: manual de utilização rápida. UA Editora.

Teixes, F. (2015). Gamificación: fundamentos y aplicaciones (Vol. 7). Editorial UOC.

Torrijos-Fincias, P., Martín-Izard, J. F. y Rodríguez-Conde, M. J. (2018). La educación emocional en la formación permanente del profesorado no universitario. Profesorado, Revista de Currículum y Formación del Profesorado, 22(1), 579-597.

Usán Supervía, P. y Salavera Bordás, C. (2018). Motivación escolar, inteligencia emocional y rendimiento académico en estudiantes de educación secundaria obligatoria. Actualidades en Psicologia, 32(125), 95-112.

Vercher Ferrándiz, M. L. (2019). La gestión de la inteligencia emocional como competencia distintiva en la docencia online para la mejora de la gestión del proceso enseñanza-aprendiraje en el ámbito de las ciencias sociales. (Tesis Doctoral). Universidad politécnica de Valencia. https://doi.org/10.4995/Thesis/10251/124817 